\title{
Oscillations of differential equations with non-monotone deviating arguments
}

George E. Chatzarakis ${ }^{1}$, Irena Jadlovská ${ }^{2}$ and Tongxing Lij ${ }^{*}$

"Correspondence: litongx2007@163.com

${ }^{3}$ School of Control Science and Engineering, Shandong University, Jinan, P.R. China

Full list of author information is available at the end of the article

\begin{abstract}
The oscillatory behavior of the solutions to a differential equation with several non-monotone arguments and nonnegative coefficients is studied, and some new oscillation criteria are given. More precisely, sufficient conditions in terms of lim sup and lim inf are established, which essentially improve several known criteria existing in the literature. The results are illustrated by examples numerically solved in MATLAB.

MSC: $34 \mathrm{~K} 06 ; 34 \mathrm{~K} 11$

Keywords: Differential equation; Non-monotone argument; Oscillatory solution; Nonoscillatory solution
\end{abstract}

\section{Introduction}

Consider the first-order linear differential equation with several variable deviating arguments of either delayed (DDE)

$$
x^{\prime}(t)+\sum_{i=1}^{m} p_{i}(t) x\left(\tau_{i}(t)\right)=0, \quad t \geq t_{0}
$$

or advanced type (ADE)

$$
x^{\prime}(t)-\sum_{i=1}^{m} q_{i}(t) x\left(\sigma_{i}(t)\right)=0, \quad t \geq t_{0}
$$

where $p_{i}, q_{i}, 1 \leq i \leq m$, are functions of nonnegative real numbers, and $\tau_{i}, \sigma_{i}, 1 \leq i \leq m$, are functions of positive real numbers satisfying

$$
\tau_{i}(t)<t, \quad t \geq t_{0} \quad \text { and } \quad \lim _{t \rightarrow \infty} \tau_{i}(t)=\infty, \quad 1 \leq i \leq m,
$$

and

$$
\sigma_{i}(t)>t, \quad t \geq t_{0}, \quad 1 \leq i \leq m,
$$

respectively.

By a solution of $(E)$ or $\left(E^{\prime}\right)$ we mean a function absolutely continuous on $\left[t_{0}, \infty\right)$ satisfying $(E)$ or $\left(E^{\prime}\right)$ for almost all $t \geq t_{0}$.

\section{Springer}


A solution of $(E)$ or $\left(E^{\prime}\right)$ is oscillatory if it is neither eventually positive nor eventually negative. If there exists an eventually positive or eventually negative solution, then the equation is nonoscillatory. An equation is oscillatory if all its solutions oscillate.

The problem of establishing sufficient conditions for the oscillation of all solutions of equations $(E)$ or $\left(E^{\prime}\right)$ has been studied extensively. The reader is referred to [1-23] and the references cited therein. Most of this work though involves the special case where the arguments are nondecreasing, while only a small fraction of these papers deal with the general case where the arguments are not necessarily monotone. See, for example, $[1-7$, $13,14]$ and the references cited therein. The general case is significant not only from the mathematical standpoint, but for its applications in the real world as well. This is because there are always natural disturbances, for example, noise in communication systems, that affect all the parameters of the equation, and therefore the fair (from a mathematical point of view) monotone arguments become non-monotone almost always.

The objective of this paper is to derive new sufficient conditions for all solutions of $(E)$ and $\left(E^{\prime}\right)$ to be oscillatory when the arguments are not necessarily monotone. Our results essentially improve several known criteria existing in the literature, which are briefly reviewed below for the reader's convenience.

Throughout this paper, we are going to use the following notation:

$$
\begin{array}{ll}
P(t):=\sum_{i=1}^{m} p_{i}(t), & \alpha:=\liminf _{t \rightarrow \infty} \int_{\tau(t)}^{t} P(s) d s, \\
Q(t):=\sum_{i=1}^{m} q_{i}(t), & \beta:=\liminf _{t \rightarrow \infty} \int_{t}^{\sigma(t)} Q(s) d s,
\end{array}
$$

and

$$
D(\omega):= \begin{cases}0, & \text { if } \omega>1 / e, \\ \frac{1-\omega-\sqrt{1-2 \omega-\omega^{2}}}{2}, & \text { if } \omega \in[0,1 / e]\end{cases}
$$

where $\tau(t):=\max _{1 \leq i \leq m}\left\{\tau_{i}(t)\right\}$ and $\sigma(t):=\min _{1 \leq i \leq m}\left\{\sigma_{i}(t)\right\}$.

\subsection{DDEs (chronological review)}

It follows from Remark 2.7.3 in [20] that if $\tau_{i}, 1 \leq i \leq m$, are nondecreasing and

$$
\limsup _{t \rightarrow \infty} \int_{\tau(t)}^{t} P(s) d s>1
$$

then every solution of $(E)$ oscillates. This result is similar to Theorem 2.1.3 [20] which is a special case of the result by Ladas, Lakshmikantham, and Papadakis [17].

In 1978, Ladde [19] and in 1982, Ladas and Stavroulakis [18] proved that if

$$
\alpha>\frac{1}{e}
$$

then every solution of $(E)$ oscillates. 
In 1984, Hunt and Yorke [11] proved that if $\tau_{i}$ are nondecreasing, $t-\tau_{i}(t) \leq \tau_{0}, 1 \leq i \leq m$, and

$$
\liminf _{t \rightarrow \infty} \sum_{i=1}^{m} p_{i}(t)\left(t-\tau_{i}(t)\right)>\frac{1}{e}
$$

then every solution of $(E)$ oscillates.

Suppose that $\tau_{i}, 1 \leq i \leq m$, are not necessarily monotone. Set

$$
h_{i}(t):=\sup _{t_{0} \leq s \leq t} \tau_{i}(s) \quad \text { and } \quad h(t):=\max _{1 \leq i \leq m} h_{i}(t), \quad t \geq t_{0}
$$

Clearly, $h_{i}, h$ are nondecreasing and $\tau_{i}(t) \leq h_{i}(t) \leq h(t)<t$ for all $t \geq t_{0}$.

In 2016, Braverman, Chatzarakis, and Stavroulakis [1] proved that if, for some $r \in \mathbb{N}$,

$$
\limsup _{t \rightarrow \infty} \int_{h(t)}^{t} \sum_{i=1}^{m} p_{i}(\zeta) a_{r}\left(h(t), \tau_{i}(\zeta)\right) d \zeta>1
$$

or

$$
\liminf _{t \rightarrow \infty} \int_{h(t)}^{t} \sum_{i=1}^{m} p_{i}(\zeta) a_{r}\left(h(t), \tau_{i}(\zeta)\right) d \zeta>\frac{1}{e}
$$

where

$$
\begin{aligned}
& a_{1}(t, s):=\exp \left\{\int_{s}^{t} P(\zeta) d \zeta\right\}, \\
& a_{r+1}(t, s):=\exp \left\{\int_{s}^{t} \sum_{i=1}^{m} p_{i}(\zeta) a_{r}\left(\zeta, \tau_{i}(\zeta)\right) d \zeta\right\},
\end{aligned}
$$

then every solution of $(E)$ oscillates.

Several improvements have been made to the above conditions, see $[3-5,7]$ to arrive at the recent forms [4]

$$
\begin{aligned}
& \limsup _{t \rightarrow \infty} \int_{h(t)}^{t} P(s) \exp \left(\int_{\tau(s)}^{h(t)} P(u) \exp \left(\int_{\tau(u)}^{u} W_{\ell}(\xi) d \xi\right) d u\right) d s>1, \\
& \limsup _{t \rightarrow \infty} \int_{h(t)}^{t} P(s) \exp \left(\int_{\tau(s)}^{h(t)} P(u) \exp \left(\int_{\tau(u)}^{u} W_{\ell}(\xi) d \xi\right) d u\right) d s>1-D(\alpha), \\
& \liminf _{t \rightarrow \infty} \int_{h(t)}^{t} P(s) \exp \left(\int_{\tau(s)}^{h(s)} P(u) \exp \left(\int_{\tau(u)}^{u} W_{\ell}(\xi) d \xi\right) d u\right) d s>\frac{1}{e},
\end{aligned}
$$

where

$$
W_{\ell}(t):=P(t)\left[1+\int_{\tau(t)}^{t} P(s) \exp \left(\int_{\tau(s)}^{t} P(u) \exp \left(\int_{\tau(u)}^{u} W_{\ell-1}(\xi) d \xi\right) d u\right) d s\right]
$$

with

$$
W_{0}(t):=P(t)\left[1+\int_{\tau(t)}^{t} P(s) \exp \left(\int_{\tau(s)}^{t} P(\omega) \exp \left(\lambda_{0} \int_{\tau(\omega)}^{\omega} P(u) d u\right) d \omega\right) d s\right],
$$

where $\lambda_{0}$ is the smaller root of the transcendental equation $\lambda=e^{\alpha \lambda}$. 


\subsection{ADEs (chronological review)}

For $\left(E^{\prime}\right)$, the dual condition of (1.3) is

$$
\limsup _{t \rightarrow \infty} \int_{t}^{\sigma(t)} Q(s) d s>1
$$

where $\sigma_{i}, 1 \leq i \leq m$, are nondecreasing (see [20], paragraph 2.7).

In 1978, Ladde [19] and in 1982, Ladas and Stavroulakis [18] proved that if

$$
\beta>\frac{1}{e}
$$

then every solution of $\left(E^{\prime}\right)$ oscillates.

In 1990, Zhou [23] proved that if $\sigma_{i}(t)-t \leq \sigma_{0}, 1 \leq i \leq m$, and

$$
\liminf _{t \rightarrow \infty} \sum_{i=1}^{m} q_{i}(t)\left(\sigma_{i}(t)-t\right)>\frac{1}{e}
$$

then every solution of $\left(E^{\prime}\right)$ oscillates; see also [8, Corollary 2.6.12].

Suppose that $\sigma_{i}, 1 \leq i \leq m$, are not necessarily monotone. Set

$$
\rho_{i}(t):=\inf _{s \geq t} \sigma_{i}(s) \quad \text { and } \quad \rho(t):=\min _{1 \leq i \leq m} \rho_{i}(t), \quad t \geq t_{0}
$$

Clearly, $\rho_{i}, \rho$ are nondecreasing and $\sigma_{i}(t) \geq \rho_{i}(t) \geq \rho(t)>t$ for all $t \geq t_{0}$.

In 2016, Braverman, Chatzarakis, and Stavroulakis [1] proved that if, for some $r \in \mathbb{N}$,

$$
\limsup _{t \rightarrow \infty} \int_{t}^{\rho(t)} \sum_{i=1}^{m} q_{i}(\zeta) b_{r}\left(\rho(t), \sigma_{i}(\zeta)\right) d \zeta>1
$$

or

$$
\liminf _{t \rightarrow \infty} \int_{t}^{\rho(t)} \sum_{i=1}^{m} q_{i}(\zeta) b_{r}\left(\rho(t), \sigma_{i}(\zeta)\right) d \zeta>\frac{1}{e}
$$

where

$$
\begin{aligned}
& b_{1}(t, s):=\exp \left\{\int_{t}^{s} Q(\zeta) d \zeta\right\} \\
& b_{r+1}(t, s):=\exp \left\{\int_{t}^{s} \sum_{i=1}^{m} q_{i}(\zeta) b_{r}\left(\zeta, \sigma_{i}(\zeta)\right) d \zeta\right\}
\end{aligned}
$$

then every solution of $\left(E^{\prime}\right)$ oscillates.

Several improvements have been made to the above conditions, see $[3-5,7]$ to arrive at the recent forms [4]

$$
\limsup _{t \rightarrow \infty} \int_{t}^{\rho(t)} Q(s) \exp \left(\int_{\rho(t)}^{\sigma(s)} Q(u) \exp \left(\int_{u}^{\sigma(u)} S_{\ell}(\xi) d \xi\right) d u\right) d s>1
$$




$$
\begin{aligned}
& \limsup _{t \rightarrow \infty} \int_{t}^{\rho(t)} Q(s) \exp \left(\int_{\rho(t)}^{\sigma(s)} Q(u) \exp \left(\int_{u}^{\sigma(u)} S_{\ell}(\xi) d \xi\right) d u\right) d s>1-D(\beta), \\
& \liminf _{t \rightarrow \infty} \int_{t}^{\rho(t)} Q(s) \exp \left(\int_{\rho(s)}^{\sigma(s)} Q(u) \exp \left(\int_{u}^{\sigma(u)} S_{\ell}(\xi) d \xi\right) d u\right) d s>\frac{1}{e}
\end{aligned}
$$

where

$$
S_{\ell}(t):=Q(t)\left[1+\int_{t}^{\sigma(t)} Q(s) \exp \left(\int_{t}^{\sigma(s)} Q(u) \exp \left(\int_{u}^{\sigma(u)} S_{\ell-1}(\xi) d \xi\right) d u\right) d s\right]
$$

with

$$
S_{0}(t):=Q(t)\left[1+\int_{t}^{\sigma(t)} Q(s) \exp \left(\int_{t}^{\sigma(s)} Q(\omega) \exp \left(\lambda_{0} \int_{\omega}^{\sigma(\omega)} Q(u) d u\right) d \omega\right) d s\right]
$$

where $\lambda_{0}$ is the smaller root of the transcendental equation $\lambda=e^{\beta \lambda}$.

\section{Main results}

\subsection{DDEs}

New sufficient conditions are established for the oscillation of all solutions of $(E)$ using an iterative method. These conditions, involving lim sup and lim inf, significantly improve several results in the literature.

We now cite two lemmas which will be used to prove the main results. Their proofs are similar to those of Lemmas 2.1.1, 2.1.3, and 2.1.2 in [8], respectively.

Lemma 1 Let $h$ be as in (1.6). Then

$$
\liminf _{t \rightarrow \infty} \int_{\tau(t)}^{t} P(s) d s=\liminf _{t \rightarrow \infty} \int_{h(t)}^{t} P(s) d s
$$

Lemma 2 Let $\alpha \in(0,1 / e], x$ be an eventually positive solution of $(E)$, and $h$ be as in (1.6).

Then

$$
\liminf _{t \rightarrow \infty} \frac{x(t)}{x(h(t))} \geq D(\alpha)
$$

and

$$
\liminf _{t \rightarrow \infty} \frac{x(h(t))}{x(t)} \geq \lambda_{0}
$$

where $\lambda_{0}$ is the smaller root of the transcendental equation $\lambda=e^{\alpha \lambda}$.

On the basis of the above lemmas, we present the following results. Without loss of generality, we deal only with eventually positive solutions of $(E)$ (or $\left(E^{\prime}\right)$ ) since, under our assumptions, if $x$ is a solution, so is $-x$.

Theorem 1 Let $\alpha \in(0,1 / e]$ and $h$ be as in (1.6). Assume that, for some $\ell \in \mathbb{N}$,

$$
\limsup _{t \rightarrow \infty} \int_{h(t)}^{t} P(s) \exp \left(\int_{\tau(s)}^{h(t)} P(u) \exp \left(\int_{\tau(u)}^{u} B_{\ell}(\xi) d \xi\right) d u\right) d s>1
$$


where

$$
B_{\ell}(t):=P(t)\left[1+\int_{\tau(t)}^{t} P(s) \exp \left(\int_{\tau(s)}^{t} P(u) \exp \left(\int_{\tau(u)}^{u} B_{\ell-1}(\xi) d \xi\right) d u\right) d s\right]
$$

with

$$
\begin{aligned}
B_{0}(t):= & P(t)[1 \\
& \left.+\int_{\tau(t)}^{t} P(s) \exp \left(\int_{\tau(s)}^{t} P(y) \exp \left(\int_{\tau(y)}^{y} P(\omega) \exp \left(\lambda_{0} \int_{\tau(\omega)}^{\omega} P(u) d u\right) d \omega\right) d y\right) d s\right],
\end{aligned}
$$

and $\lambda_{0}$ is the smaller root of the transcendental equation $\lambda=e^{\alpha \lambda}$. Then every solution of (E) oscillates.

Proof Assume the opposite. Let $x$ be an eventually positive solution of $(E)$. Then there exists a $t_{1}>t_{0}$ such that $x(t)>0$ and $x\left(\tau_{i}(t)\right)>0$ for all $t \geq t_{1}$ and $1 \leq i \leq m$. Thus, by virtue of $(E)$,

$$
x^{\prime}(t)=-\sum_{i=1}^{m} p_{i}(t) x\left(\tau_{i}(t)\right) \leq 0 \quad \text { for all } t \geq t_{1}
$$

which implies that $x$ is an eventually nonincreasing function of positive numbers.

Dividing $(E)$ by $x(t)>0$ and integrating the resulting equality on $[\tau(t), t]$, we deduce that

$$
\int_{\tau(t)}^{t} \frac{x^{\prime}(u)}{x(u)} d u=-\int_{\tau(t)}^{t} \sum_{i=1}^{m} p_{i}(u) \frac{x\left(\tau_{i}(u)\right)}{x(u)} d u
$$

or

$$
\int_{\tau(t)}^{t} \frac{x^{\prime}(u)}{x(u)} d u \leq-\int_{\tau(t)}^{t}\left(\sum_{i=1}^{m} p_{i}(u)\right) \frac{x(\tau(u))}{x(u)} d u,
$$

or

$$
\int_{\tau(t)}^{t} \frac{x^{\prime}(u)}{x(u)} d u \leq-\int_{\tau(t)}^{t} P(u) \frac{x(\tau(u))}{x(u)} d u .
$$

Therefore,

$$
x(\tau(t)) \geq x(t) \exp \left(\int_{\tau(t)}^{t} P(u) \frac{x(\tau(u))}{x(u)} d u\right) .
$$

Combining $(E)$ and (2.6), we arrive at

$$
\begin{aligned}
0 & =x^{\prime}(t)+\sum_{i=1}^{m} p_{i}(t) x\left(\tau_{i}(t)\right) \geq x^{\prime}(t)+P(t) x(\tau(t)) \\
& \geq x^{\prime}(t)+P(t) x(t) \exp \left(\int_{\tau(t)}^{t} P(u) \frac{x(\tau(u))}{x(u)} d u\right) .
\end{aligned}
$$


Dividing (2.7) by $x(t)>0$ and integrating the resulting inequality on $[\tau(t), t]$, we obtain

$$
\int_{\tau(t)}^{t} \frac{x^{\prime}(\omega)}{x(\omega)} d \omega \leq-\int_{\tau(t)}^{t} P(\omega) \exp \left(\int_{\tau(\omega)}^{\omega} P(u) \frac{x(\tau(u))}{x(u)} d u\right) d \omega
$$

or

$$
x(\tau(t)) \geq x(t) \exp \left(\int_{\tau(t)}^{t} P(\omega) \exp \left(\int_{\tau(\omega)}^{\omega} P(u) \frac{x(\tau(u))}{x(u)} d u\right) d \omega\right)
$$

Combining $(E)$ and (2.8), we have

$$
x^{\prime}(t)+P(t) x(t) \exp \left(\int_{\tau(t)}^{t} P(\omega) \exp \left(\int_{\tau(\omega)}^{\omega} P(u) \frac{x(\tau(u))}{x(u)} d u\right) d \omega\right) \leq 0
$$

Dividing (2.9) by $x(t)>0$ and integrating the resulting inequality on $[\tau(s), t]$, we get

$$
\int_{\tau(s)}^{t} \frac{x^{\prime}(y)}{x(y)} d y \leq-\int_{\tau(s)}^{t} P(y) \exp \left(\int_{\tau(y)}^{y} P(\omega) \exp \left(\int_{\tau(\omega)}^{\omega} P(u) \frac{x(\tau(u))}{x(u)} d u\right) d \omega\right) d y
$$

or

$$
\begin{aligned}
& x(\tau(s)) \\
& \quad \geq x(t) \exp \left(\int_{\tau(s)}^{t} P(y) \exp \left(\int_{\tau(y)}^{y} P(\omega) \exp \left(\int_{\tau(\omega)}^{\omega} P(u) \frac{x(\tau(u))}{x(u)} d u\right) d \omega\right) d y\right) .
\end{aligned}
$$

Integrating $(E)$ from $\tau(t)$ to $t$, using (2.10), multiplying by $P(t)$, and taking into account the fact that $x^{\prime}(t) \leq-P(t) x(\tau(t))$, we conclude that

$$
\begin{aligned}
0 \geq & x^{\prime}(t)+P(t) x(t) \\
& +P(t) x(t) \int_{\tau(t)}^{t} P(s) \exp \left(\int_{\tau(s)}^{t} P(y)\right. \\
& \left.\quad \times \exp \left(\int_{\tau(y)}^{y} P(\omega) \exp \left(\int_{\tau(\omega)}^{\omega} P(u) \frac{x(\tau(u))}{x(u)} d u\right) d \omega\right) d y\right) d s .
\end{aligned}
$$

It follows from $\tau(u) \leq h(u)$ that

$$
\begin{aligned}
0 \geq & x^{\prime}(t)+P(t) x(t) \\
& +P(t) x(t) \int_{\tau(t)}^{t} P(s) \exp \left(\int_{\tau(s)}^{t} P(y)\right. \\
& \left.\times \exp \left(\int_{\tau(y)}^{y} P(\omega) \exp \left(\int_{\tau(\omega)}^{\omega} P(u) \frac{x(h(u))}{x(u)} d u\right) d \omega\right) d y\right) d s .
\end{aligned}
$$


By virtue of (2.3), for each $\epsilon>0$ and for sufficiently large $t$, the last inequality becomes

$$
\begin{aligned}
& 0 \geq x^{\prime}(t) \\
& \quad+P(t)\left[1+\int_{\tau(t)}^{t} P(s) \exp \left(\int_{\tau(s)}^{t} P(y)\right.\right. \\
& \left.\left.\quad \times \exp \left(\int_{\tau(y)}^{y} P(\omega) \exp \left(\left(\lambda_{0}-\epsilon\right) \int_{\tau(\omega)}^{\omega} P(u) d u\right) d \omega\right) d y\right) d s\right] x(t)
\end{aligned}
$$

or

$$
x^{\prime}(t)+B_{0}(t, \epsilon) x(t) \leq 0
$$

with

$$
\begin{aligned}
B_{0}(t, \epsilon):= & P(t)\left[1+\int_{\tau(t)}^{t} P(s) \exp \left(\int_{\tau(s)}^{t} P(y)\right.\right. \\
& \left.\left.\times \exp \left(\int_{\tau(y)}^{y} P(\omega) \exp \left(\left(\lambda_{0}-\epsilon\right) \int_{\tau(\omega)}^{\omega} P(u) d u\right) d \omega\right) d y\right) d s\right] .
\end{aligned}
$$

Applying the Grönwall inequality in (2.11), we obtain

$$
x(\tau(u)) \geq x(u) \exp \left(\int_{\tau(u)}^{u} B_{0}(\xi, \epsilon) d \xi\right) .
$$

Dividing $(E)$ by $x(t)>0$, integrating the resulting equality on $[\tau(s), t]$ and using $(2.12)$, we deduce that

$$
-\int_{\tau(s)}^{t} \frac{x^{\prime}(u)}{x(u)} d u \geq \int_{\tau(s)}^{t} P(u) \frac{x(\tau(u))}{x(u)} d u \geq \int_{\tau(s)}^{t} P(u) \exp \left(\int_{\tau(u)}^{u} B_{0}(\xi, \epsilon) d \xi\right) d u
$$

or

$$
x(\tau(s)) \geq x(t) \exp \left(\int_{\tau(s)}^{t} P(u) \exp \left(\int_{\tau(u)}^{u} B_{0}(\xi, \epsilon) d \xi\right) d u\right) .
$$

Integrating $(E)$ from $\tau(t)$ to $t$ and using (2.13), we obtain

$$
x(t)-x(\tau(t))+x(t) \int_{\tau(t)}^{t} P(s) \exp \left(\int_{\tau(s)}^{t} P(u) \exp \left(\int_{\tau(u)}^{u} B_{0}(\xi, \epsilon) d \xi\right) d u\right) d s \leq 0 .
$$

Multiplying the last inequality by $P(t)$, we have

$$
\begin{aligned}
& P(t) x(t)-P(t) x(\tau(t))+P(t) x(t) \int_{\tau(t)}^{t} P(s) \exp \left(\int_{\tau(s)}^{t} P(u) \exp \left(\int_{\tau(u)}^{u} B_{0}(\xi, \epsilon) d \xi\right) d u\right) d s \\
& \quad \leq 0
\end{aligned}
$$

which, in view of $x^{\prime}(t) \leq-P(t) x(\tau(t))$, reduces to

$$
x^{\prime}(t)+P(t) x(t)+P(t) x(t) \int_{\tau(t)}^{t} P(s) \exp \left(\int_{\tau(s)}^{t} P(u) \exp \left(\int_{\tau(u)}^{u} B_{0}(\xi, \epsilon) d \xi\right) d u\right) d s \leq 0 .
$$


Hence, for sufficiently large $t$,

$$
x^{\prime}(t)+P(t)\left[1+\int_{\tau(t)}^{t} P(s) \exp \left(\int_{\tau(s)}^{t} P(u) \exp \left(\int_{\tau(u)}^{u} B_{0}(\xi, \epsilon) d \xi\right) d u\right) d s\right] x(t) \leq 0
$$

or

$$
x^{\prime}(t)+B_{1}(t, \epsilon) x(t) \leq 0,
$$

where

$$
B_{1}(t, \epsilon):=P(t)\left[1+\int_{\tau(t)}^{t} P(s) \exp \left(\int_{\tau(s)}^{t} P(u) \exp \left(\int_{\tau(u)}^{u} B_{0}(\xi, \epsilon) d \xi\right) d u\right) d s\right] .
$$

Following the above procedures, we can inductively construct the inequalities

$$
x^{\prime}(t)+B_{\ell}(t, \epsilon) x(t) \leq 0, \quad \ell \in \mathbb{N},
$$

where

$$
B_{\ell}(t, \epsilon):=P(t)\left[1+\int_{\tau(t)}^{t} P(s) \exp \left(\int_{\tau(s)}^{t} P(u) \exp \left(\int_{\tau(u)}^{u} B_{\ell-1}(\xi, \epsilon) d \xi\right) d u\right) d s\right]
$$

and

$$
x(\tau(s)) \geq x(h(t)) \exp \left(\int_{\tau(s)}^{h(t)} P(u) \exp \left(\int_{\tau(u)}^{u} B_{\ell}(\xi, \epsilon) d \xi\right) d u\right) .
$$

Integrating $(E)$ from $h(t)$ to $t$ and using (2.14), we arrive at

$$
\begin{aligned}
& x(t)-x(h(t))+x(h(t)) \int_{h(t)}^{t} P(s) \exp \left(\int_{\tau(s)}^{h(t)} P(u) \exp \left(\int_{\tau(u)}^{u} B_{\ell}(\xi, \epsilon) d \xi\right) d u\right) d s \\
& \quad \leq 0 .
\end{aligned}
$$

If we omit $x(t)>0$, then (2.15) becomes

$$
x(h(t))\left[\int_{h(t)}^{t} P(s) \exp \left(\int_{\tau(s)}^{h(t)} P(u) \exp \left(\int_{\tau(u)}^{u} B_{\ell}(\xi, \epsilon) d \xi\right) d u\right) d s-1\right]<0,
$$

which means that

$$
\limsup _{t \rightarrow \infty} \int_{h(t)}^{t} P(s) \exp \left(\int_{\tau(s)}^{h(t)} P(u) \exp \left(\int_{\tau(u)}^{u} B_{\ell}(\xi, \epsilon) d \xi\right) d u\right) d s \leq 1 .
$$

This inequality contradicts (2.4) due to the fact that $\epsilon$ may be taken arbitrarily small. The proof is complete.

Theorem 2 Let $\alpha \in(0,1 / e]$ and $h$ be as in (1.6). If, for some $\ell \in \mathbb{N}$,

$$
\limsup _{t \rightarrow \infty} \int_{h(t)}^{t} P(s) \exp \left(\int_{\tau(s)}^{h(t)} P(u) \exp \left(\int_{\tau(u)}^{u} B_{\ell}(\xi) d \xi\right) d u\right) d s>1-D(\alpha),
$$

where $B_{\ell}$ is as in (2.5), then every solution of $(E)$ oscillates. 
Proof Let $x$ be an eventually positive solution of $(E)$. As in the proof of Theorem 1, (2.15) holds, that is,

$$
x(t)-x(h(t))+x(h(t)) \int_{h(t)}^{t} P(s) \exp \left(\int_{\tau(s)}^{h(t)} P(u) \exp \left(\int_{\tau(u)}^{u} B_{\ell}(\xi, \epsilon) d \xi\right) d u\right) d s \leq 0,
$$

which yields

$$
\int_{h(t)}^{t} P(s) \exp \left(\int_{\tau(s)}^{h(t)} P(u) \exp \left(\int_{\tau(u)}^{u} B_{\ell}(\xi, \epsilon) d \xi\right) d u\right) d s \leq 1-\frac{x(t)}{x(h(t))},
$$

and hence

$$
\begin{aligned}
& \limsup _{t \rightarrow \infty} \int_{h(t)}^{t} P(s) \exp \left(\int_{\tau(s)}^{h(t)} P(u) \exp \left(\int_{\tau(u)}^{u} B_{\ell}(\xi, \epsilon) d \xi\right) d u\right) d s \\
& \quad \leq 1-\liminf _{t \rightarrow \infty} \frac{x(t)}{x(h(t))} .
\end{aligned}
$$

It follows from Lemma 2 that inequality (2.2) is satisfied. So, by virtue of (2.2) and (2.17),

$$
\limsup _{t \rightarrow \infty} \int_{h(t)}^{t} P(s) \exp \left(\int_{\tau(s)}^{h(t)} P(u) \exp \left(\int_{\tau(u)}^{u} B_{\ell}(\xi, \epsilon) d \xi\right) d u\right) d s \leq 1-D(\alpha) .
$$

This inequality contradicts (2.16) due to the fact that $\epsilon$ may be taken arbitrarily small. The proof is complete.

Theorem 3 Let $\alpha \in(0,1 / e]$ and $h$ be as in (1.6). If, for some $\ell \in \mathbb{N}$,

$$
\liminf _{t \rightarrow \infty} \int_{h(t)}^{t} P(s) \exp \left(\int_{\tau(s)}^{h(s)} P(u) \exp \left(\int_{\tau(u)}^{u} B_{\ell}(\xi) d \xi\right) d u\right) d s>\frac{1}{e}
$$

where $B_{\ell}$ is as in (2.5), then every solution of $(E)$ oscillates.

Proof As above, assume that $(E)$ has a nonoscillatory solution $x$ which is eventually positive. Then there exists a $t_{1}>t_{0}$ such that $x(t)>0, x\left(\tau_{i}(t)\right)>0,1 \leq i \leq m$, for all $t \geq t_{1}$. Thus, by $(E)$, we have

$$
x^{\prime}(t)=-\sum_{i=1}^{m} p_{i}(t) x\left(\tau_{i}(t)\right) \leq 0 \quad \text { for all } t \geq t_{1}
$$

which implies that $x$ is an eventually nonincreasing function of positive numbers. Moreover, as in the proof of Theorem 1, (2.14) is fulfilled.

Dividing $(E)$ by $x(t)$ and integrating the resulting equality from $h(t)$ to $t$, we deduce that

$$
\begin{aligned}
\ln \left(\frac{x(h(t))}{x(t)}\right) & =\int_{h(t)}^{t} \sum_{i=1}^{m} p_{i}(s) \frac{x\left(\tau_{i}(s)\right)}{x(s)} d s \\
& \geq \int_{h(t)}^{t}\left(\sum_{i=1}^{m} p_{i}(s)\right) \frac{x(\tau(s))}{x(s)} d s=\int_{h(t)}^{t} P(s) \frac{x(\tau(s))}{x(s)} d s
\end{aligned}
$$




$$
\begin{aligned}
& \geq \int_{h(t)}^{t} P(s) \frac{x(h(s))}{x(s)} \exp \left(\int_{\tau(s)}^{h(s)} P(u) \exp \left(\int_{\tau(u)}^{u} B_{\ell}(\xi, \epsilon) d \xi\right) d u\right) d s \\
& \geq \int_{h(t)}^{t} P(s) \exp \left(\int_{\tau(s)}^{h(s)} P(u) \exp \left(\int_{\tau(u)}^{u} B_{\ell}(\xi, \epsilon) d \xi\right) d u\right) d s .
\end{aligned}
$$

It follows from (2.18) that there exists a constant $c>0$ such that, for all sufficiently large $t$,

$$
\int_{h(t)}^{t} P(s) \exp \left(\int_{\tau(s)}^{h(s)} P(u) \exp \left(\int_{\tau(u)}^{u} B_{\ell}(\xi) d \xi\right) d u\right) d s \geq c>\frac{1}{e} .
$$

Choose $c^{\prime}$ such that $c>c^{\prime}>1 / e$. For every $\epsilon>0$ satisfying $c-\epsilon>c^{\prime}$, we get

$$
\int_{h(t)}^{t} P(s) \exp \left(\int_{\tau(s)}^{h(s)} P(u) \exp \left(\int_{\tau(u)}^{u} B_{\ell}(\xi, \epsilon) d \xi\right) d u\right) d s \geq c-\epsilon>c^{\prime}>\frac{1}{e} .
$$

Hence, we derive from (2.19) and (2.20) that

$$
\ln \left(\frac{x(h(t))}{x(t)}\right) \geq c^{\prime}
$$

which yields

$$
\frac{x(h(t))}{x(t)} \geq e^{c^{\prime}} \geq e c^{\prime}>1
$$

and so

$$
x(h(t)) \geq\left(e c^{\prime}\right) x(t)
$$

Repeating the above steps, it follows by induction that, for any positive integer $k$,

$$
\frac{x(h(t))}{x(t)} \geq\left(e c^{\prime}\right)^{k} \quad \text { for sufficiently large } t
$$

Since $e c^{\prime}>1$, there is a $k \in \mathbb{N}$ satisfying $k>2\left(\ln 2-\ln c^{\prime}\right) /\left(1+\ln c^{\prime}\right)$ such that, for $t$ sufficiently large,

$$
\frac{x(h(t))}{x(t)} \geq\left(e c^{\prime}\right)^{k}>\left(\frac{2}{c^{\prime}}\right)^{2}
$$

Taking the integral on $[h(t), t]$, which is not less than $c^{\prime}$, we split the interval into two parts where integrals are not less than $c^{\prime} / 2$, let $t_{m} \in(h(t), t)$ be the splitting point:

$$
\begin{aligned}
& \int_{h(t)}^{t_{m}} P(s) \exp \left(\int_{\tau(s)}^{h(s)} P(u) \exp \left(\int_{\tau(u)}^{u} B_{\ell}(\xi, \epsilon) d \xi\right) d u\right) d s \geq \frac{c^{\prime}}{2} \\
& \int_{t_{m}}^{t} P(s) \exp \left(\int_{\tau(s)}^{h(s)} P(u) \exp \left(\int_{\tau(u)}^{u} B_{\ell}(\xi, \epsilon) d \xi\right) d u\right) d s \geq \frac{c^{\prime}}{2}
\end{aligned}
$$


Integrating $(E)$ from $t_{m}$ to $t$, we arrive at

$$
\begin{aligned}
0 & =x(t)-x\left(t_{m}\right)+\int_{t_{m}}^{t} \sum_{i=1}^{m} p_{i}(s) x\left(\tau_{i}(s)\right) d s \\
& \geq x(t)-x\left(t_{m}\right)+\int_{t_{m}}^{t} P(s) x(\tau(s)) d s \\
& \geq x(t)-x\left(t_{m}\right)+x(h(t)) \int_{t_{m}}^{t} P(s) \exp \left(\int_{\tau(s)}^{h(s)} P(u) \exp \left(\int_{\tau(u)}^{u} B_{\ell}(\xi, \epsilon) d \xi\right) d u\right) d s \\
& >-x\left(t_{m}\right)+x(h(t)) \int_{t_{m}}^{t} P(s) \exp \left(\int_{\tau(s)}^{h(s)} P(u) \exp \left(\int_{\tau(u)}^{u} B_{\ell}(\xi, \epsilon) d \xi\right) d u\right) d s .
\end{aligned}
$$

This, along with the second inequality in (2.22), implies that

$$
x\left(t_{m}\right)>\frac{c^{\prime}}{2} x(h(t))
$$

Similarly, integrating $(E)$ from $h(t)$ to $t_{m}$ and subsequently applying (2.14), we conclude that

$$
x\left(t_{m}\right)-x(h(t))+x\left(h\left(t_{m}\right)\right) \int_{h(t)}^{t_{m}} P(s) \exp \left(\int_{\tau(s)}^{h(s)} P(u) \exp \left(\int_{\tau(u)}^{u} B_{\ell}(\xi, \epsilon) d \xi\right) d u\right) d s \leq 0 .
$$

If we omit $x\left(t_{m}\right)>0$ in the left-hand side, then the strict inequality becomes valid:

$$
-x(h(t))+x\left(h\left(t_{m}\right)\right) \int_{h(t)}^{t_{m}} P(s) \exp \left(\int_{\tau(s)}^{h(s)} P(u) \exp \left(\int_{\tau(u)}^{u} B_{\ell}(\xi, \epsilon) d \xi\right) d u\right) d s<0 .
$$

This, along with the first inequality in (2.22), yields

$$
x(h(t))>\frac{c^{\prime}}{2} x\left(h\left(t_{m}\right)\right) .
$$

Combining inequalities (2.23) and (2.24), we obtain

$$
x\left(h\left(t_{m}\right)\right)<\frac{2}{c^{\prime}} x(h(t))<\left(\frac{2}{c^{\prime}}\right)^{2} x\left(t_{m}\right),
$$

which contradicts (2.21). This completes the proof.

\subsection{ADEs}

Similar oscillation criteria can easily be derived for the (dual) advanced differential equation $\left(E^{\prime}\right)$. The proofs are omitted due to the fact that they are quite similar to those of the delay equation.

Theorem 4 Let $\beta \in(0,1 / e]$ and $\rho$ be as in (1.15). If, for some $\ell \in \mathbb{N}$,

$$
\limsup _{t \rightarrow \infty} \int_{t}^{\rho(t)} Q(s) \exp \left(\int_{\rho(t)}^{\sigma(s)} Q(u) \exp \left(\int_{u}^{\sigma(u)} \Phi_{\ell}(\xi) d \xi\right) d u\right) d s>1
$$


where

$$
\Phi_{\ell}(t):=Q(t)\left[1+\int_{t}^{\sigma(t)} Q(s) \exp \left(\int_{t}^{\sigma(s)} Q(u) \exp \left(\int_{u}^{\sigma(u)} \Phi_{\ell-1}(\xi) d \xi\right) d u\right) d s\right]
$$

with

$$
\begin{aligned}
\Phi_{0}(t):= & Q(t)\left[1+\int_{t}^{\sigma(t)} Q(s) \exp \left(\int_{t}^{\sigma(s)} Q(y)\right.\right. \\
& \left.\left.\times \exp \left(\int_{y}^{\sigma(y)} Q(\omega) \exp \left(\lambda_{0} \int_{\omega}^{\sigma(\omega)} Q(u) d u\right) d \omega\right) d y\right) d s\right],
\end{aligned}
$$

and $\lambda_{0}$ is the smaller root of the transcendental equation $\lambda=e^{\beta \lambda}$, then every solution of $\left(E^{\prime}\right)$ oscillates.

Theorem 5 Let $\beta \in(0,1 / e]$ and $\rho$ be as in (1.15). If, for some $\ell \in \mathbb{N}$,

$$
\limsup _{t \rightarrow \infty} \int_{t}^{\rho(t)} Q(s) \exp \left(\int_{\rho(t)}^{\sigma(s)} Q(u) \exp \left(\int_{u}^{\sigma(u)} \Phi_{\ell}(\xi) d \xi\right) d u\right) d s>1-D(\beta),
$$

where $\Phi_{\ell}$ is as in (2.26), then every solution of $\left(E^{\prime}\right)$ oscillates.

Theorem 6 Let $\beta \in(0,1 / e]$ and $\rho$ be as in (1.15). If, for some $\ell \in \mathbb{N}$,

$$
\liminf _{t \rightarrow \infty} \int_{t}^{\rho(t)} Q(s) \exp \left(\int_{\rho(s)}^{\sigma(s)} Q(u) \exp \left(\int_{u}^{\sigma(u)} \Phi_{\ell}(\xi) d \xi\right) d u\right) d s>\frac{1}{e},
$$

where $\Phi_{\ell}$ is as in (2.26), then every solution of $\left(E^{\prime}\right)$ oscillates.

\section{Examples}

The examples provided in this section illustrate cases where the results of the present paper ensure oscillations, whereas the previously known results fail to apply in these equations. Moreover, these examples show the improvement attained by the obtained conditions over the known ones. The calculations performed have been produced in MATLAB.

Example 1 Consider the DDE

$$
x^{\prime}(t)+\frac{523}{6250} x\left(\tau_{1}(t)\right)+\frac{523}{25,000} x\left(\tau_{2}(t)\right)=0, \quad t \geq 0,
$$

with (see Fig. 1(a))

$$
\tau_{1}(t)=\left\{\begin{array}{ll}
-t+12 k-2, & \text { if } t \in[6 k, 6 k+1], \\
4 t-18 k-7, & \text { if } t \in[6 k+1,6 k+2], \\
0.5 t+3 k, & \text { if } t \in[6 k+2,6 k+4], \\
-6 t+42 k+26, & \text { if } t \in[6 k+4,6 k+5], \\
8 t-42 k-44, & \text { if } t \in[6 k+5,6 k+6],
\end{array} \quad \text { and } \quad \tau_{2}(t)=\tau_{1}(t)-0.5,\right.
$$

where $k \in \mathbb{N}_{0}$ and $\mathbb{N}_{0}$ is the set containing all nonnegative integers. 

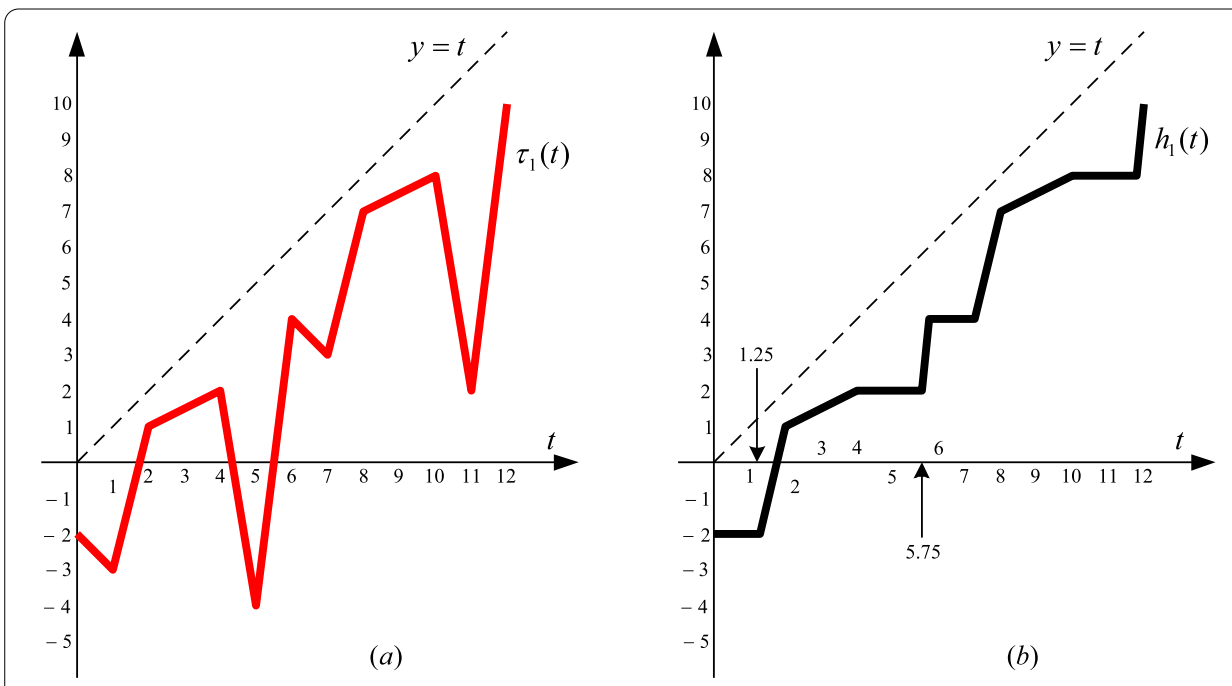

Figure 1 The graphs of $\tau_{1}(t)$ and $h_{1}(t)$

By (1.6), we see (Fig. 1(b)) that

$$
h_{1}(t)=\left\{\begin{array}{ll}
6 k-2, & \text { if } t \in[6 k, 6 k+1.25] \\
4 t-18 k-7, & \text { if } t \in[6 k+1.25,6 k+2], \\
0.5 t+3 k, & \text { if } t \in[6 k+2,6 k+4], \\
6 k+2, & \text { if } t \in[6 k+4,6 k+5.75], \\
8 t-42 k-44, & \text { if } t \in[6 k+5.75,6 k+6]
\end{array} \text { and } h_{2}(t)=h_{1}(t)-0.5,\right.
$$

and consequently,

$$
h(t)=\max _{1 \leq i \leq 2}\left\{h_{i}(t)\right\}=h_{1}(t) \quad \text { and } \quad \tau(t)=\max _{1 \leq i \leq 2} \tau_{i}(t)=\tau_{1}(t) .
$$

It is easy to see that

$$
\alpha=\liminf _{t \rightarrow \infty} \int_{\tau(t)}^{t} \sum_{i=1}^{2} p_{i}(s) d s=\liminf _{k \rightarrow \infty} \int_{6 k+1}^{6 k+2}\left(\frac{523}{6250}+\frac{523}{25,000}\right) d s=0.1046
$$

and therefore, the smaller root of $e^{0.1046 \lambda}=\lambda$ is $\lambda_{0}=1.12486$.

Observe that the function $F_{\ell}: \mathbb{R}_{0} \rightarrow \mathbb{R}_{+}$defined as

$$
F_{\ell}(t)=\int_{h(t)}^{t} P(s) \exp \left(\int_{\tau(s)}^{h(t)} P(u) \exp \left(\int_{\tau(u)}^{u} B_{\ell}(\xi) d \xi\right) d u\right) d s
$$

attains its maximum at $t=6 k+5.75, k \in \mathbb{N}_{0}$, for every $\ell \in \mathbb{N}$. In particular,

$$
F_{1}(t=6 k+5.75)=\int_{6 k+2}^{6 k+5.75} P(s) \exp \left(\int_{\tau(s)}^{6 k+2} P(u) \exp \left(\int_{\tau(u)}^{u} B_{1}(\xi) d \xi\right) d u\right) d s \simeq 1.043
$$


and thus

$$
\limsup _{t \rightarrow \infty} F_{1}(t) \simeq 1.043>1 .
$$

That is, condition (2.4) of Theorem 1 is satisfied for $\ell=1$, which implies that every solution of (3.1) oscillates.

Observe, however, that

$$
\begin{aligned}
& \limsup _{k \rightarrow \infty} \int_{6 k+2}^{6 k+5.75}\left(\frac{523}{6250}+\frac{523}{25,000}\right) d s=0.39225<1, \\
& \alpha=0.1046<\frac{1}{e},
\end{aligned}
$$

and

$$
\begin{aligned}
& \liminf _{t \rightarrow \infty} \sum_{i=1}^{2} p_{i}(t)\left(t-\tau_{i}(t)\right) \\
& =\liminf _{t \rightarrow \infty}\left[\frac{523}{6250}\left(t-\tau_{1}(t)\right)+\frac{523}{25,000}\left(t-\left(\tau_{1}(t)-0.5\right)\right)\right] \\
& =\liminf _{t \rightarrow \infty}\left[\frac{523}{5000}\left(t-\tau_{1}(t)\right)+\frac{523}{50,000}\right] \\
& =\liminf _{t \rightarrow \infty}\left[\frac{523}{5000}\left(t-\tau_{1}(t)\right)\right]+\frac{523}{50,000} \\
& =\frac{523}{5000} \cdot \liminf _{t \rightarrow \infty}\left(t-\tau_{1}(t)\right)+\frac{523}{50,000} \\
& \quad=\frac{523}{5000} \cdot 1+\frac{523}{50,000}=0.11506<\frac{1}{e} .
\end{aligned}
$$

Note that the function $D_{r}: \mathbb{R}_{0} \rightarrow \mathbb{R}_{+}$defined as

$$
D_{r}(t)=\int_{h(t)}^{t} \sum_{i=1}^{2} p_{i}(\zeta) a_{r}\left(h(t), \tau_{i}(\zeta)\right) d \zeta
$$

attains its maximum at $t=6 k+5.75$ and its minimum at $t=6 k+2, k \in \mathbb{N}_{0}$, for every $r \in \mathbb{N}$. In particular,

$$
\begin{aligned}
D_{1}(t=6 k+5.75)= & \int_{6 k+2}^{6 k+5.75} \sum_{i=1}^{2} p_{i}(\zeta) a_{1}\left(6 k+2, \tau_{i}(\zeta)\right) d \zeta \\
= & \int_{6 k+2}^{6 k+4}\left[p_{1}(\zeta) a_{1}\left(6 k+2, \tau_{1}(\zeta)\right)+p_{2}(\zeta) a_{1}\left(6 k+2, \tau_{2}(\zeta)\right)\right] d \zeta \\
& +\int_{6 k+4}^{6 k+5}\left[p_{1}(\zeta) a_{1}\left(6 k+2, \tau_{1}(\zeta)\right)+p_{2}(\zeta) a_{1}\left(6 k+2, \tau_{2}(\zeta)\right)\right] d \zeta \\
& +\int_{6 k+5}^{6 k+5.75}\left[p_{1}(\zeta) a_{1}\left(6 k+2, \tau_{1}(\zeta)\right)+p_{2}(\zeta) a_{1}\left(6 k+2, \tau_{2}(\zeta)\right)\right] d \zeta \\
\simeq & 0.4803,
\end{aligned}
$$




$$
\begin{aligned}
D_{1}(t=6 k+2) & =\int_{6 k+1}^{6 k+2} \sum_{i=1}^{2} p_{i}(\zeta) a_{1}\left(6 k+1, \tau_{i}(\zeta)\right) d \zeta \\
& =\int_{6 k+1}^{6 k+2}\left[p_{1}(\zeta) a_{1}\left(6 k+1, \tau_{1}(\zeta)\right)+p_{2}(\zeta) a_{1}\left(6 k+1, \tau_{2}(\zeta)\right)\right] d \zeta \\
& \simeq 0.1313 .
\end{aligned}
$$

Hence,

$$
\begin{aligned}
& \limsup _{t \rightarrow \infty} D_{1}(t) \simeq 0.4803<1, \\
& \liminf _{t \rightarrow \infty} D_{1}(t) \simeq 0.1313<1 / e .
\end{aligned}
$$

Finally, using algorithms on MATLAB software, we obtain

$$
\begin{aligned}
& \limsup _{t \rightarrow \infty} \int_{h(t)}^{t} P(s) \exp \left(\int_{\tau(s)}^{h(t)} P(u) \exp \left(\int_{\tau(u)}^{u} W_{1}(\xi) d \xi\right) d u\right) d s \simeq 0.8157<1, \\
& 0.8157<1-D(\alpha) \simeq 0.9938 \\
& \liminf _{t \rightarrow \infty} \int_{h(t)}^{t} P(s) \exp \left(\int_{\tau(s)}^{h(s)} P(u) \exp \left(\int_{\tau(u)}^{u} W_{1}(\xi) d \xi\right) d u\right) d s \simeq 0.1064<\frac{1}{e} .
\end{aligned}
$$

That is, none of conditions (1.3)-(1.5), (1.7) and (1.8) (for $r=1)$, and (1.9)-(1.11) (for $\ell=1)$ is satisfied.

Comments It is worth noticing that the improvement of condition (2.4) over the corresponding condition (1.3) is significant, approximately $165.9 \%$ when comparing the values on the left-hand side of these conditions. Meanwhile, the improvement compared to conditions (1.7) and (1.9) is very satisfactory, around $117.16 \%$ and $27.87 \%$, respectively. In addition, observe that conditions (1.7)-(1.11) do not ensure oscillations from the first iteration. On the contrary, condition (2.4) is fulfilled from the first iteration. This means that this condition is better and more efficient, leading to oscillations much faster than conditions (1.7)-(1.11).

Example 2 Consider the ADE

$$
x^{\prime}(t)-\frac{417}{5000} x\left(\sigma_{1}(t)\right)-\frac{139}{2500} x\left(\sigma_{2}(t)\right)=0, \quad t \geq 0,
$$

with (see Fig. 2(a))

$$
\sigma_{1}(t)=\left\{\begin{array}{ll}
5 k+2, & \text { if } t \in[5 k, 5 k+1], \\
5 t-20 k-3, & \text { if } t \in[5 k+1,5 k+2], \\
-2 t+15 k+11, & \text { if } t \in[5 k+2,5 k+3], \\
3 t-10 k-4, & \text { if } t \in[5 k+3,5 k+4], \\
-t+10 k+12, & \text { if } t \in[5 k+4,5 k+5],
\end{array} \text { and } \quad \sigma_{2}(t)=\sigma_{1}(t)+0.5,\right.
$$

where $k \in \mathbb{N}_{0}$ and $\mathbb{N}_{0}$ stands for the set containing all nonnegative integers. 


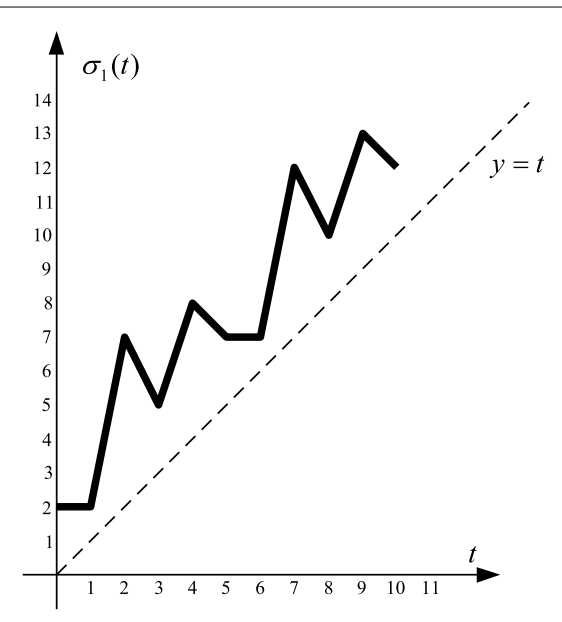

(a)

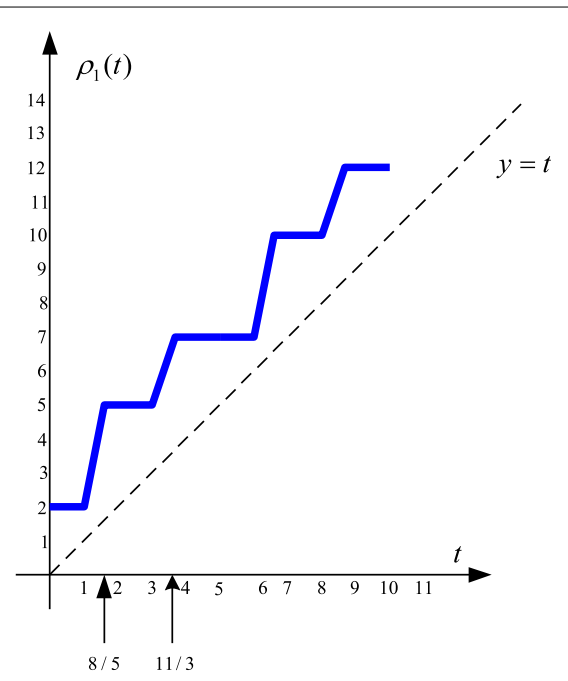

(b)

Figure 2 The graphs of $\sigma_{1}(t)$ and $\rho_{1}(t)$

By (1.15), we see (Fig. 2(b)) that

$$
\rho_{1}(t)=\left\{\begin{array}{ll}
5 k+2, & \text { if } t \in[5 k, 5 k+1], \\
5 t-20 k-3, & \text { if } t \in[5 k+1,5 k+8 / 5], \\
5 k+5, & \text { if } t \in[5 k+8 / 5,5 k+3], \\
3 t-10 k-4, & \text { if } t \in[5 k+3,5 k+11 / 3], \\
5 k+7, & \text { if } t \in[5 k+11 / 3,5 k+5],
\end{array} \text { and } \quad \rho_{2}(t)=\rho_{1}(t)+0.5,\right.
$$

and consequently,

$$
\rho(t)=\min _{1 \leq i \leq 2}\left\{\rho_{i}(t)\right\}=\rho_{1}(t) \quad \text { and } \quad \sigma(t)=\min _{1 \leq i \leq 2}\left\{\sigma_{i}(t)\right\}=\sigma_{1}(t) .
$$

It is easy to see that

$$
\beta=\liminf _{t \rightarrow \infty} \int_{t}^{\sigma(t)} \sum_{i=1}^{2} q_{i}(s) d s=\liminf _{k \rightarrow \infty} \int_{5 k+1}^{5 k+2} \frac{139}{1000} d s=0.139
$$

and therefore, the smaller root of $e^{0.139 \lambda}=\lambda$ is $\lambda_{0}=1.17789$.

Observe that the function $F_{\ell}: \mathbb{R}_{0} \rightarrow \mathbb{R}_{+}$defined as

$$
F_{\ell}(t)=\int_{t}^{\rho(t)} Q(s) \exp \left(\int_{\rho(t)}^{\sigma(s)} Q(u) \exp \left(\int_{u}^{\sigma(u)} \Phi_{\ell}(\xi) d \xi\right) d u\right) d s
$$

attains its maximum at $t=5 k+8 / 5, k \in \mathbb{N}_{0}$, for every $\ell \in \mathbb{N}$. In particular, by using an algorithm on MATLAB software, we obtain

$$
F_{1}(t=5 k+8 / 5)=\int_{5 k+8 / 5}^{5 k+5} Q(s) \exp \left(\int_{5 k+2}^{\sigma(s)} Q(u) \exp \left(\int_{u}^{\sigma(u)} \Phi_{1}(\xi) d \xi\right) d u\right) d s \simeq 0.9996
$$


and so

$$
\liminf _{t \rightarrow \infty} F_{1}(t) \simeq 0.9996>1-D(\beta) \simeq 0.9886 .
$$

That is, condition (2.27) of Theorem 5 is satisfied for $\ell=1$, and therefore every solution of (3.2) oscillates.

Observe, however, that

$$
\begin{aligned}
\limsup _{k \rightarrow \infty} \int_{5 k+8 / 5}^{5 k+5} \frac{139}{1000} d s= & 0.4726<1, \\
\beta=0.139<\frac{1}{e}, & \\
\liminf _{t \rightarrow \infty} \sum_{i=1}^{2} q_{i}(t)\left(\sigma_{i}(t)-t\right) & =\liminf _{t \rightarrow \infty} \sum_{i=1}^{2} q_{i}(t)\left(\rho_{i}(t)-t\right) \\
& =\liminf _{t \rightarrow \infty}\left[\frac{417}{5000}\left(\rho_{1}(t)-t\right)+\frac{139}{2500}\left(\rho_{1}(t)+0.5-t\right)\right] \\
& =\liminf _{t \rightarrow \infty}\left[0.139\left(\rho_{1}(t)-t\right)+0.0278\right] \\
& =\liminf _{t \rightarrow \infty}\left[0.139\left(\rho_{1}(t)-t\right)\right]+0.0278 \\
& =0.139 \cdot 1+0.0278=0.1668<\frac{1}{e},
\end{aligned}
$$

$$
\begin{aligned}
& \limsup _{t \rightarrow \infty} \int_{t}^{\rho(t)} \sum_{i=1}^{2} q_{i}(\zeta) b_{1}\left(\rho(t), \sigma_{i}(\zeta)\right) d \zeta \\
& \quad \limsup _{k \rightarrow \infty} \int_{5 k+8 / 5}^{5 k+5} \sum_{i=1}^{2} q_{i}(\zeta) b_{1}\left(5 k+5, \sigma_{i}(\zeta)\right) d \zeta \\
& \quad \limsup _{k \rightarrow \infty}\left\{\begin{array}{r}
\int_{5 k+8 / 5}^{5 k+2}\left[q_{1}(\zeta) b_{1}\left(5 k+5, \sigma_{1}(\zeta)\right)+q_{2}(\zeta) b_{1}\left(5 k+5, \sigma_{2}(\zeta)\right)\right] d \zeta \\
+\int_{5 k+3}^{5 k+3}\left[q_{1}(\zeta) b_{1}\left(5 k+5, \sigma_{1}(\zeta)\right)+q_{2}(\zeta) b_{1}\left(5 k+5, \sigma_{2}(\zeta)\right)\right] d \zeta \\
+\int_{5 k+4}^{5 k+4}\left[q_{1}(\zeta) b_{1}\left(5 k+5, \sigma_{1}(\zeta)\right)+q_{2}(\zeta) b_{1}\left(5 k+5, \sigma_{2}(\zeta)\right)\right] d \zeta \\
+\int_{5 k+4}^{5 k+5}\left[q_{1}(\zeta) b_{1}\left(5 k+5, \sigma_{1}(\zeta)\right)+q_{2}(\zeta) b_{1}\left(5 k+5, \sigma_{2}(\zeta)\right)\right] d \zeta
\end{array}\right\} \\
& =0.6108<1,
\end{aligned}
$$

and

$$
\begin{aligned}
& \liminf _{t \rightarrow \infty} \int_{t}^{\rho(t)} \sum_{i=1}^{2} q_{i}(\zeta) b_{1}\left(\rho(t), \sigma_{i}(\zeta)\right) d \zeta \\
& \quad=\liminf _{k \rightarrow \infty} \int_{5 k+1}^{5 k+2}\left[q_{1}(\zeta) b_{1}\left(5 k+2, \sigma_{1}(\zeta)\right)+q_{2}(\zeta) b_{1}\left(5 k+2, \sigma_{2}(\zeta)\right)\right] d \zeta \\
& \simeq 0.2065<1 / e .
\end{aligned}
$$

That is, none of conditions (1.12)-(1.14), (1.16), and (1.17) (for $r=1$ ) is satisfied.

Finally, by using algorithms on MATLAB software, we see that none of conditions (1.18)-(1.20) (for $\ell=1$ ) is satisfied. 
Remark 1 Similarly, one can present examples to illustrate the efficiency of other main results.

\begin{abstract}
Acknowledgements
The authors express their sincere gratitude to the editors and two anonymous referees for the careful reading of the original manuscript and useful comments that helped to improve the presentation of the results and accentuate important details.
\end{abstract}

\title{
Funding
}

This research is supported by NNSF of P.R. China (Grant No. 61503171), CPSF (Grant No. 2015M582091), and NSF of Shandong Province (Grant No. ZR2016JL021). The research of the second author is supported by the grant project KEGA 035TUKE-4/2017.

\section{Availability of data and materials}

Data sharing not applicable to this article as no datasets were generated or analysed during the current study.

\section{Competing interests}

The authors declare that they have no competing interests.

\section{Authors' contributions}

All three authors contributed equally to this work. They all read and approved the final version of the manuscript.

\section{Author details}

'Department of Electrical and Electronic Engineering Educators, School of Pedagogical and Technological Education (ASPETE), Athens, Greece. ${ }^{2}$ Department of Mathematics and Theoretical Informatics, Faculty of Electrical Engineering and Informatics, Technical University of Košice, Košice, Slovakia. ${ }^{3}$ School of Control Science and Engineering, Shandong University, Jinan, P.R. China.

\section{Publisher's Note}

Springer Nature remains neutral with regard to jurisdictional claims in published maps and institutional affiliations.

Received: 8 April 2019 Accepted: 30 May 2019 Published online: 14 June 2019

\section{References}

1. Braverman, E., Chatzarakis, G.E., Stavroulakis, I.P.: Iterative oscillation tests for differential equations with several non-monotone arguments. Adv. Differ. Equ. 2016, 87 (2016)

2. Braverman, E., Karpuz, B.: On oscillation of differential and difference equations with non-monotone delays. Appl. Math. Comput. 218(7), 3880-3887 (2011)

3. Chatzarakis, G.E.: Oscillations caused by several non-monotone deviating arguments. Differ. Equ. Appl. 9(3), 285-310 (2017)

4. Chatzarakis, G.E.: Oscillations of equations caused by several deviating arguments. Opusc. Math. 39(3), 321-353 (2019)

5. Chatzarakis, G.E., Li, T.: Oscillations of differential equations generated by several deviating arguments. Adv. Differ. Equ. 2017, 292 (2017)

6. Chatzarakis, G.E., Li, T.: Oscillation criteria for delay and advanced differential equations with nonmonotone arguments. Complexity 2018, Article ID 8237634 (2018)

7. Chatzarakis, G.E., Péics, H.: Differential equations with several non-monotone arguments: an oscillation result. Appl. Math. Lett. 68, 20-26 (2017)

8. Erbe, L.H., Kong, Q., Zhang, B.G.: Oscillation Theory for Functional Differential Equations. Dekker, New York (1995)

9. Erbe, L.H., Zhang, B.G.: Oscillation for first order linear differential equations with deviating arguments. Differ. Integral Equ. 1(3), 305-314 (1988)

10. Fukagai, N., Kusano, T.: Oscillation theory of first order functional differential equations with deviating arguments, Ann. Mat. Pura Appl. (4) 136(1), 95-117 (1984)

11. Hunt, B.R., Yorke, J.A.: When all solutions of $x^{\prime}(t)=-\sum q_{i}(t) x\left(t-T_{i}(t)\right)$ oscillate. J. Differ. Equ. 53(2), 139-145 (1984)

12. Koplatadze, R.G., Chanturiya, T.A.: Oscillating and monotone solutions of first-order differential equations with deviating argument. Differ. Uravn. 18(8), 1463-1465, 1472 (1982) (in Russian)

13. Koplatadze, R., Kvinikadze, G.: On the oscillation of solutions of first order delay differential inequalities and equations. Georgian Math. J. 1(6), 675-685 (1994)

14. Kulenović, M.R., Grammatikopoulos, M.K.: First order functional differential inequalities with oscillating coefficients. Nonlinear Anal. 8(9), 1043-1054 (1984)

15. Kusano, T: On even-order functional differential equations with advanced and retarded arguments. J. Differ. Equ. 45(1), 75-84 (1982)

16. Kwong, M.K.: Oscillation of first-order delay equations. J. Math. Anal. Appl. 156(1), 274-286 (1991)

17. Ladas, G., Lakshmikantham, V., Papadakis, J.S.: Oscillations of higher-order retarded differential equations generated by the retarded argument. In: Delay and Functional Differential Equations and Their Applications, pp. 219-231. Academic Press, New York (1972)

18. Ladas, G., Stavroulakis, I.P.: Oscillations caused by several retarded and advanced arguments. J. Differ. Equ. 44(1), 134-152 (1982) 
19. Ladde, G.S.: Oscillations caused by retarded perturbations of first order linear ordinary differential equations. Atti Accad. Naz. Lincei, Rend. Cl. Sci. Fis. Mat. Nat. 63(5), 351-359 (1977)

20. Ladde, G.S., Lakshmikantham, V., Zhang, B.G.: Oscillation Theory of Differential Equations with Deviating Arguments. Monographs and Textbooks in Pure and Applied Mathematics, vol. 110. Dekker, New York (1987)

21. Yu, J.S., Wang, Z.C., Zhang, B.G., Qian, X.Z.: Oscillations of differential equations with deviating arguments. Panam. Math. J. 2(2), 59-78 (1992)

22. Zhang, B.G.: Oscillation of the solutions of the first-order advanced type differential equations. Sci. Explor. 2(3), 79-82 (1982)

23. Zhou, D.: On some problems on oscillation of functional differential equations of first order. J. Shandong Univ. Nat. Sci. 25(4), 434-442 (1990)

Submit your manuscript to a SpringerOpen ${ }^{\circ}$ journal and benefit from:

- Convenient online submission

- Rigorous peer review

Open access: articles freely available online

- High visibility within the field

- Retaining the copyright to your article

Submit your next manuscript at $\mathbf{s p r i n g e r o p e n . c o m ~}$ 